\title{
Juvenile polyposis syndrome and hereditary haemorrhagic telangiectasia caused by the SMAD4 gene mutation in a paediatric patient - a case report and a review of the literature
}

\author{
Dominika Kaps-Kopiec ${ }^{1}$, Andrzej Pławski ${ }^{2,3}$, Magdalena Badura-Stronka4 ${ }^{4}$ Mikołaj Teisseyre', \\ Maciej Dądalski' , Joanna Cielecka-Kuszyk' ${ }^{5}$, Joanna Pawłowska' \\ 'Department of Gastroenterology, Hepatology, Nutritional Disorders and Pediatrics, Children's Memorial Health Institute in \\ Warsaw, Poland \\ 2Institute of Human Genetics, Polish Academy of Sciences, Poznan, Poland \\ ${ }^{3}$ Department of General, Endocrine Surgery and Gastroenterological Oncology, Poznan University of Medical Science, Poland \\ ${ }^{4}$ Department of Medical Genetics, Poznan University of Medical Sciences, Poland \\ ${ }^{5}$ Department of Pathology, Children's Memorial Health Institute in Warsaw
}

\section{ABSTRACT}

Germline mutations in the SMAD4 gene cause juvenile polyposis syndrome and hereditary haemorrhagic telangiectasia. In some cases, the coexistence of these 2 syndromes can be observed among probands with the mutation in the SMAD4 gene. A combined syndrome of juvenile polyps of the gastrointestinal tract and hereditary haemorrhagic telangiectasia caused by mutations of the SMAD4 gene is recognized as a distinct disease entity (OMIM \#175050). Herein we present a case of a teenage boy with an affected family history, who was admitted to our department in order to broaden the diagnosis of the causes of his rectal bleeding and recurrent nasal bleeding. In the course of investigation polyps in his gastrointestinal tract and arteriovenous malformations in his lungs were revealed.

KEY WORDS:

cancer, polyps, telangiectasia, organ arteriovenous malformations.

\section{INTRODUCTION}

Juvenile polyposis syndrome (JPS) and hereditary haemorrhagic telangiectasia (HHT) are 2 distinct autosomal diseases. However, in patients with mutations in the SMAD4 gene (Mothers against decapentaplegic homologue 4 gene), they can be present as a combined syndrome. The coexistence of these diseases was first described early in the 1980s. Nevertheless, the association between this combined syndrome and mutation in the SMAD4 gene was first reported in 2004 by Gallione et al. [1]. Empirical evidence indicates that patients with
JPS-HHT are at risk of an early onset of colorectal cancer (mean age 28 years) [2].

Juvenile polyposis syndrome is an autosomal dominant disorder, characterized by the predisposition to hamartomatous (juvenile) polyps in the gastrointestinal tract, especially in the stomach, small intestine, colon, and rectum. The term "juvenile" refers to the histopathology of the polyp and not to the age of the onset of polyps. The prevalence rate of the JPS ranges from 1 in 160,000 to 1 in 100,000 people [3]. Rectal bleeding is often its primary manifestation and may appear in early childhood. It is worth mentioning that this condition

\section{ADDRESS FOR CORRESPONDENCE:}

Dominika Kaps-Kopiec, Department of Gastroenterology, Hepatology, Nutritional Disorders and Pediatrics,

Children’s Memorial Health Institute in Warsaw, Poland, e-mail: d.kaps-kopiec@ipczd.pl 
is associated with an increased risk of gastrointestinal tract cancer, with a lifetime risk of $38 \%$ to $68 \%$ [3]. The most likely cancer is that of the colon, but cancers of the stomach, upper gastrointestinal tract, and pancreas have been reported.

Hereditary haemorrhagic telangiectasia is also an autosomal dominant disorder characterized by vascular dysplasia with mucocutaneus telangiectasia and organ arteriovenous malformations (AVMs) mainly affecting the lungs, liver, and brain. It was originally known as Osler-Weber-Rendu syndrome after the 3 scientists who described it at the turn of the 20th century.

The aim of the study is to present the medical case of a teenage boy with combined juvenile polyposis syndrome and hereditary haemorrhagic telangiectasia.

\section{CASE REPORT}

An 11-year-old boy was referred to the regional Gastroenterology Department in 2014 because of rec-
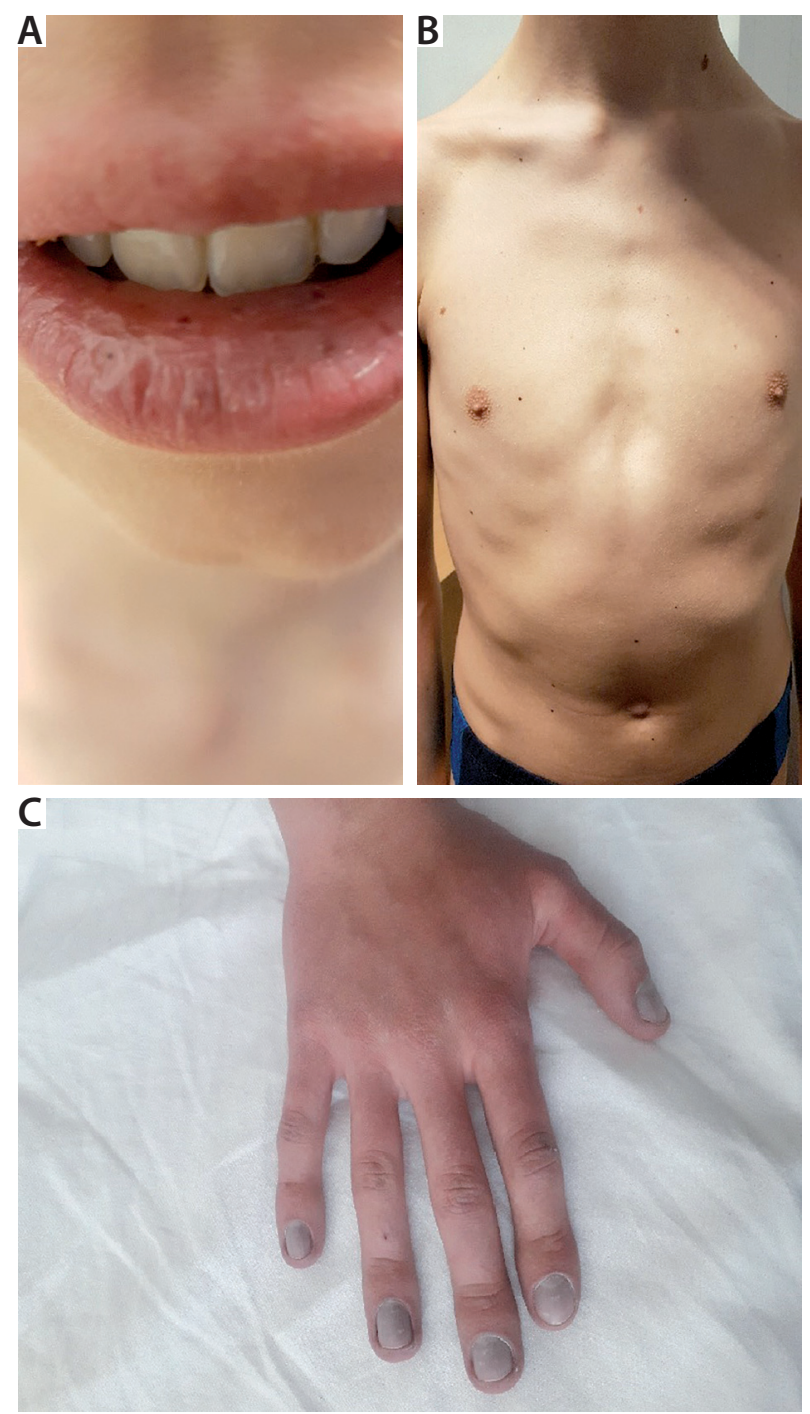

FIGURE 1. Signs revealed in the physical examination: A) telangiectasia on bottom lip; B) funnel-shaped chest; C) cyanosis of nails, clubbed fingers tal bleeding. The rectal bleeding had been noticed for the first time 7 years earlier and was most frequent after defecation. The patient also complained of recurrent nasal bleeding. The boy's mother underwent a colectomy in adulthood because of polyposis syndrome. She suffers from arteriovenous malformations in the lungs.

During the physical examination on admission, asthenic body type, protruding shoulder blades, and skin discoloration on his left forearm were noticed. Laboratory tests revealed elevated inflammation parameters (CRP $14.2 \mathrm{mg} / \mathrm{dl}$, normal range: $0.1-10.0 \mathrm{mg} / \mathrm{l}$ ) and reduced capillary blood saturation (83.1\%). The abdominal ultrasound examination was normal. During hospitalization, an endoscopy was performed and showed oesophageal erosions, a sentinel polyp in the stomach, a $1-\mathrm{cm}$ polyp in the duodenum, a $1-\mathrm{cm}$ polyp in the ascending colon, and six $2-4-\mathrm{cm}$ polyps in the sigmoid colon and rectum. Histology revealed hyperplastic polyps. The patient was directed to our Department.

A clinical interview revealed periodical stools with a small amount of blood (3-4 times a month). In the physical examination small telangiectasias on the lower lip, a funnel-shaped chest, hypermobility of interphalangeal joints in the hands, arachnodactyly, and cyanosis of the lips and nails were noticed (Fig. 1).

Endoscopy revealed over a dozen polyps in the large intestine, the biggest aggregation in the rectum. Eight polyps were removed, the biggest being $1.5 \mathrm{~cm}$ in diameter. Histopathology described juvenile polyposis with a strong inflammation infiltrate.

A control colonoscopy was carried out 2 months later. During the examination 18 polyps were removed. Because of the desaturation during anaesthesia, the patient was directed to the Department of Pulmonology. CT angiography of chest was carried out and revealed arteriovenous malformations in the lungs. Home oxygen therapy was recommended and caused a rise in the oxygen saturation from $85 \%$ to $92 \%$.

The patient was subsequently admitted to the Cardiology Department. Echocardiography showed a dilatation of the pulmonary artery to $2 \mathrm{~cm}$ and small tricuspid valve regurgitation. Diagnostic cardiac catheterization was carried out and showed numerous arteriovenous malformations, but their seclusion was infeasible because of the anatomy. Lung transplantation was considered. Sildenafil therapy, among other things, was introduced to the pharmacological treatment, and an improvement in effort tolerance was achieved. The patient could abstain from home oxygen therapy.

The patient underwent a gastroscopy and colonoscopy once a year (on average). Due to the diagnosis of the JPS, molecular genetic tests were conducted to exclude mutations in the BMRP1A (bone morphogenetic protein receptor type 1 A), SMAD4, and PTEN (phosphatase 

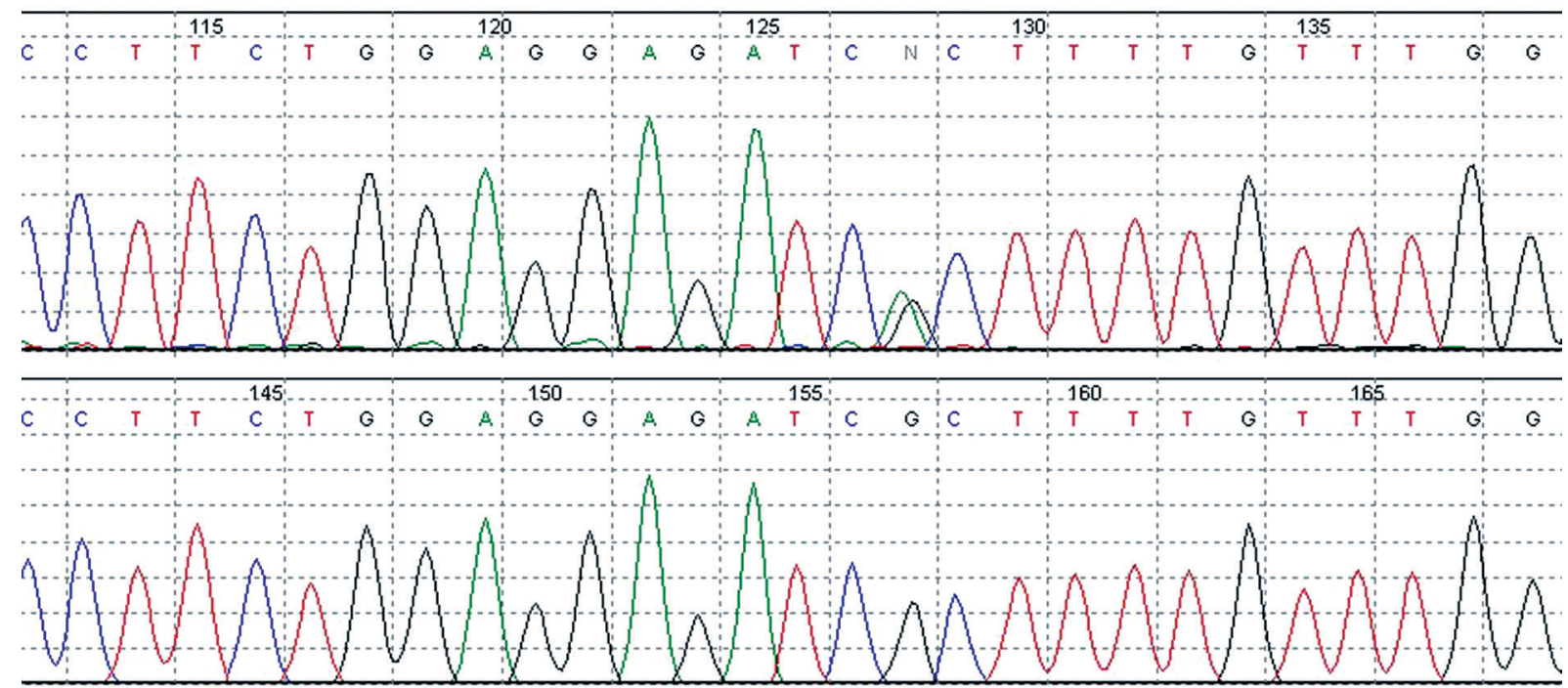

FIGURE 2. Sanger sequencing of SMAD4 gene fragment A patient, B control, arrow indicates c.1082G>A, p.Arg361His
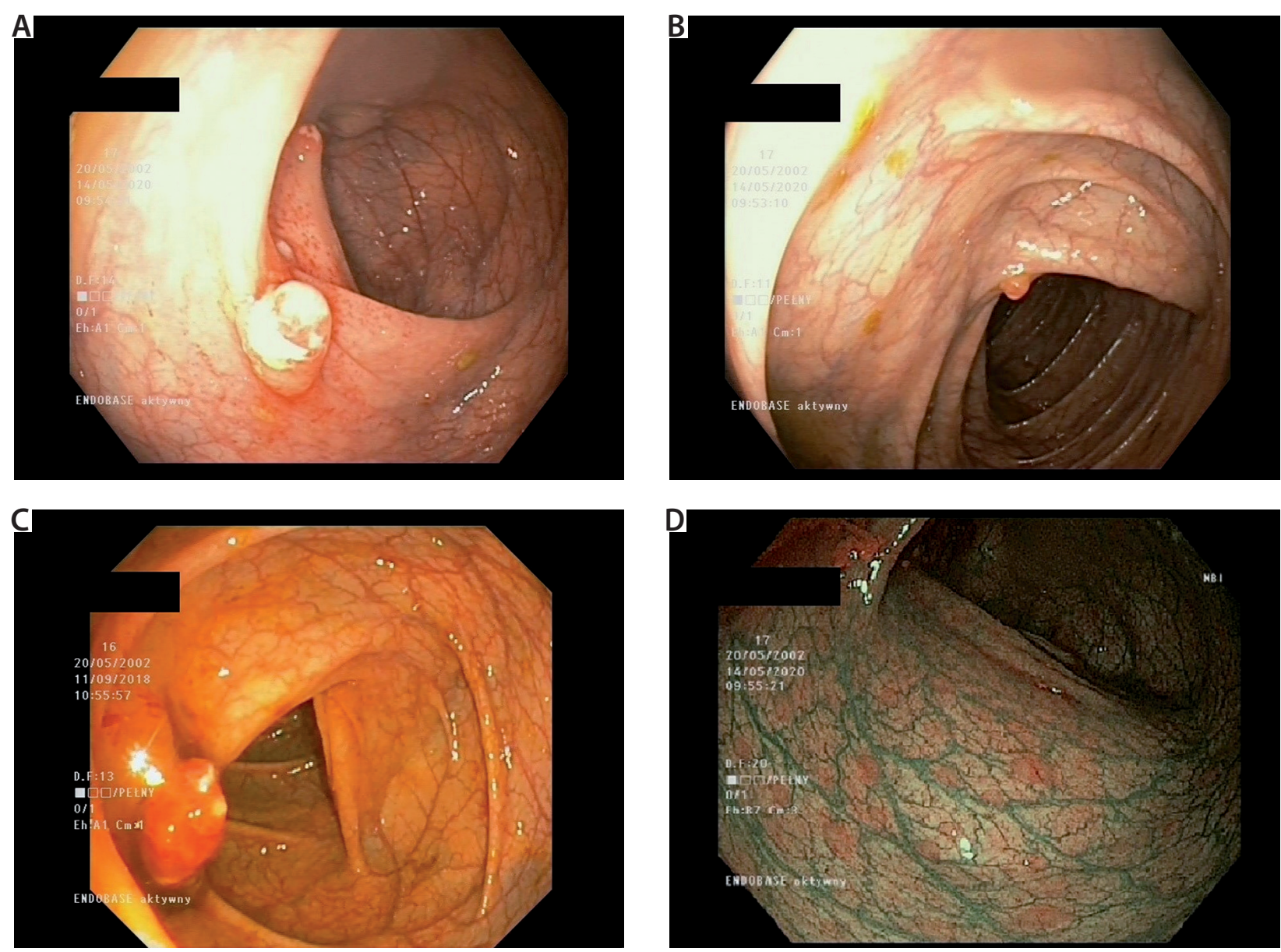

FIGURE 3. Changes revealed during colonoscopy

and tensin homologue) genes. Sanger sequencing of the SMAD4 gene identified a pathogenic substitution c.1082G >A (p. Arg361His, Fig. 2).

The last endoscopy showed esophagitis LA B and numerous sitting and pedunculated polyps in the colon (mostly in the proximal colon, with a diameter of 4 to $10 \mathrm{~mm}$, displayed in Fig. 3). The histopathology revealed juvenile polyps (presented in Fig. 4). Magnetic resonance imaging (MRI) of the brain was carried out and did not show any malformations. An ultrasound examination also did not reveal any arteriovenous malformations in the liver.

Because of the SMAD4 mutation and higher risk of colorectal cancer and the fact that the burden of polyps is not manageable by the polypectomy alone, a colectomy should be considered in future. 

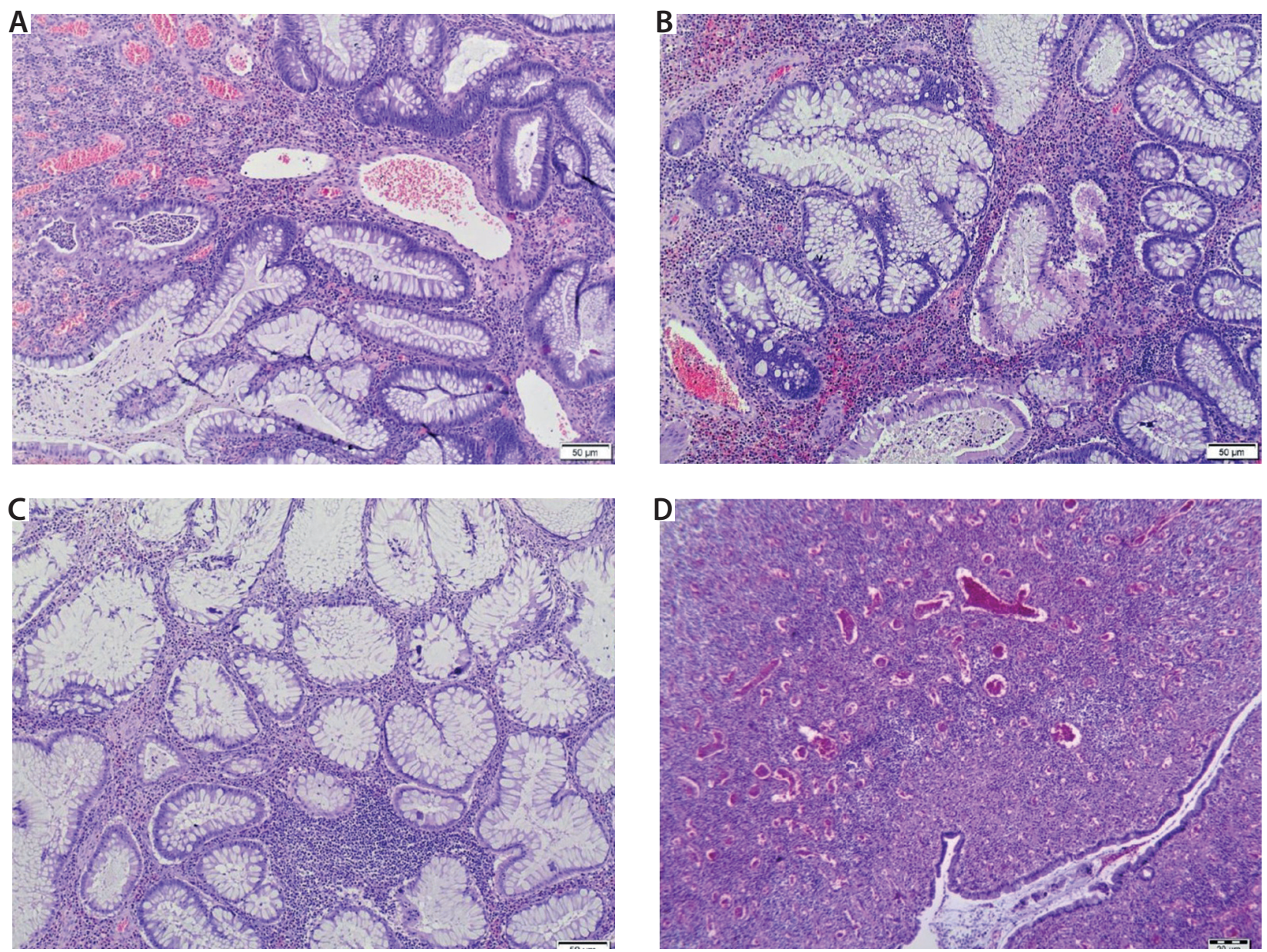

FIGURE 4. Microscopic changes in the juvenile polyp. Hematoxylin and eosin staining. A) cystically dilated glands, inflammatory infiltrates into the glands, B) and () glandular hyperplastic changes; D) stromal vascular reaction

The patient's younger brother also had a gastroscopy and colonoscopy. The first examination showed only a small, creating polyp in rectum. The histopathology revealed inflammation changes. An examination, which took place 3 years later, did not show any changes. Molecular genetic tests were also conducted, but they did not identify mutations in the BMRP1A, SMAD4, and PTEN genes; notably, the mutation c.1082G>A was not found.

\section{DISCUSSION}

The mutation in the SMAD4 gene causes a combined syndrome of juvenile polyps of the gastrointestinal tract and HHT, also known as JP-HHT. It is a very rare condition, and it can have various clinical presentations. Juvenile polyposis (JP) can be present in infancy and in adulthood with abdominal pain, anaemia, acute or chronic gastrointestinal bleeding, prolapsed rectal polyps, bowel obstruction, or intussusception [2]. Patients may also be asymptomatic [2]. The diagnostic criteria of JPS proposed by Jass et al. [4] contain 5 or more juvenile polyps of the colon or rectum, or juvenile polyps in other parts of the gastrointestinal tract, or any number of juvenile polyps and a positive family history. In $40 \%$ to $60 \%$ of patients with JPS a mutation in SMAD 4 (18q21.1) or BMPR1A (10q23.2) genes is reported [3]. It is worth remembering that many patients with JPS due to SMAD4 mutation have features of HHT (epistaxis, telangiectasia, clubbing, and AVM) [5]. The most common feature of HHT is epistaxis. The arteriovenous malformations can lead to a life-threatening haemorrhage, circulatory insufficiency, brain abscesses, and stroke. Eighty per cent of families suffering from HHT have a mutation in the endoglin $(E N G)$ or activin receptor-like kinase 1

TABLE 1. The Curacao criteria for HHT [7]

\begin{tabular}{|l|l|}
\hline 1. & Epistaxis - spontaneous, recurrent nose bleeds \\
\hline 2. & Telangiectases - multiple, at characteristic sites: lips, oral cavity, fingers, nose \\
\hline 3. & $\begin{array}{l}\text { Visceral lesions such as gastrointestinal telangiectasia (with or without bleeding), pulmonary AVM, hepatic AVM, cerebral AVMs, } \\
\text { spinal AVM }\end{array}$ \\
\hline 4. & Family history - a first-degree relative with HHT according to these criteria \\
\hline
\end{tabular}


$(A L K 1)$ genes. Twenty per cent of patients have a mutation in the SMAD4 gene or in the new loci mapped to chromosome 5 and chromosome 7. It is known that HHT patients with SMAD4 mutations have anaemia more frequently than do patients with alterations of the ENG and ALK1 gene [2].

Clinical features of HHT in patients with SMAD4 mutations [6] are epistaxis, mucocutaneus telangiectasias, AVM (visceral, hepatic, intracranial), aortopathy, and intrapulmonary shunting on echocardiogram.

The Curacao criteria for HHT [7] is definite if 3 criteria are present, possible or suspected if 2 criteria are present, and unlikely if fewer than 2 criteria are present (Table 1).

The first symptom, as in the case of our patient, can be hypoxia. Children with HHT are at risk of life-threatening complications. In JPS patients with mutations within the SMAD4 gene the risk of colorectal cancer is higher (in a cohort of 14 SMAD4 Danish patients 3 colorectal cancers occurred at a mean age of 34 years) [3]; the risk of severe gastric polyposis and gastric cancer appears to be increased [3]. Thoracic aortic disease and mitral dysfunction have also been reported [6]. That is why systematic HHT screening is recommended for JPS patients with proven SMAD4 mutation.

A Position Paper From the ESPGHAN Polyposis Working Group recommends also that a colectomy should only be discussed in patients in whom the burden of polyps is not manageable by polypectomy alone, and/ or leading to uncontrollable anaemia or hypoalbuminaemia from colonic polyposis [3]. The question is, however, whether the recommendation for polypectomy should not be expanded for patients with JPS and SMAD4 mutations and JPS-HHT overlap syndrome in which the risk of colorectal cancer is higher.

Jelsig et al. [8] described the clinical characteristics of 14 Danish patients with SMAD4 mutations. They found that probands with SMAD4 mutation have symptoms of both HHT and JPS and that the frequency of PAVM and of gastric involvement with polyps in this group is higher than in patients with HHT or JPS not caused by mutations in the SMAD4 gene. Furthermore, the authors detected 4 occurrences of GI - cancer at a young age. Similar results were presented in the work of Schwenter et al., in which 10 patients met the clinical diagnostic criteria for both JP and HHT [2]. Three JPSHHT patients developed early-onset colorectal cancer [2]. In a study of 80 unrelated families with juvenile polyposis syndrome, Aretz et al. found that 35 of the cases of JPS were caused by the SMAD4 mutations, of which $22 \%$ exhibited features of the HHT [9]. All the described cases of gastric cancer in that publication (i.e. 7 cases) were reported in families with mutations in the SMAD4 gene. In addition to those studies, there are also case presentations in the literature. Teekakirikul et al. reported 2 patients with JPS-HHT and with SMAD4 mutations associated

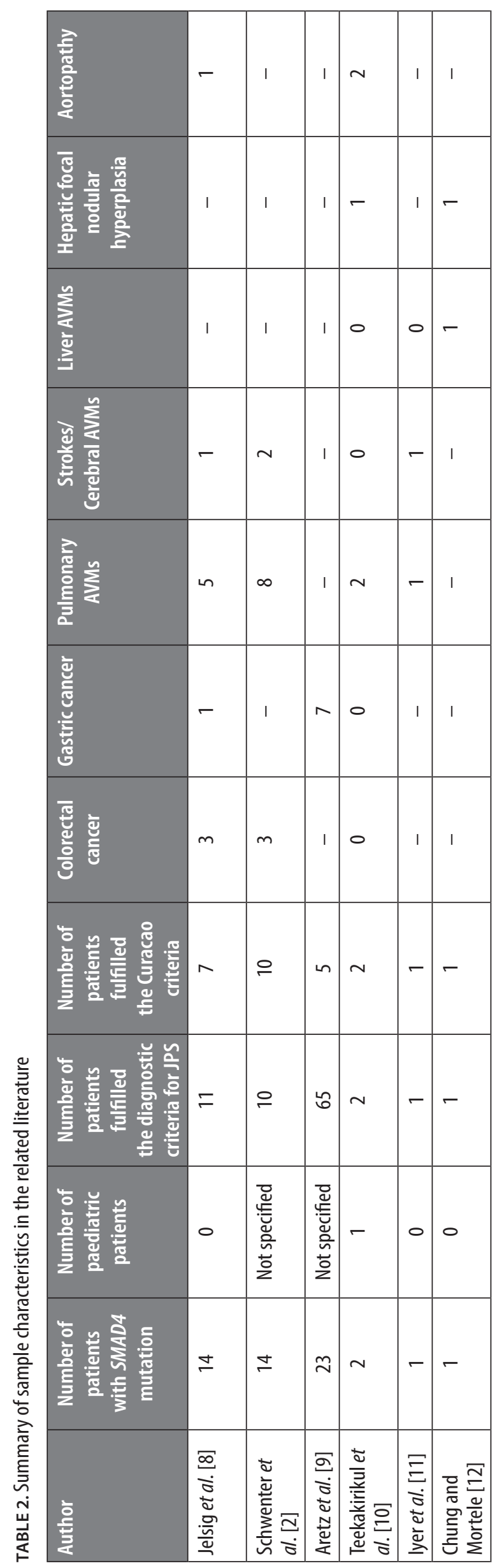


with thoracic aortic disease [10]. This report confirmed the fact that SMAD4 mutations predispose to thoracic aortic aneurysms and aortic dissections. Their work emphasized that the thoracic aorta should be screened in patients with SMAD4 mutations to prevent death from dissection [10]. Iyer et al. reported a patient with the JPSHHT syndrome and SMAD4 mutations [11]. The patient suffered from asymptomatic cerebral and pulmonary arteriovenous malformations. The only study that reports multiple arteriovenous malformations involving the liver was that of Chung et al. [12].

We found only one paper by Pantaleoni et al. describing a patient with severe hypoxaemia secondary to an inoperable pulmonary arteriovenous malformations treated by long-term management with sildenafil [13].

In Table 2 we summarize data from the literature concerning JPS-HHT syndrome.

We present the case because of the rarity of the JPSHHT syndrome in the literature, particularly in paediatric patients, and the need for multidisciplinary care of these patients to avoid life-threatening complications. We highlight a positive influence of sildenafil on the respiratory function of our patient.

\section{CONCLUSIONS}

1. Patients with features of both JP and HHT should be screened for mutations in the SMAD4 gene.

2. Paediatric patients with JPS-HHT syndrome and arteriovenous malformations of the lungs may profit from sildenafil treatment.

\section{DISCLOSURE}

The authors declare no conflict of interest.

\section{REFERENCES}

1. Gallione CJ, Repetto GM, Legius E, et al. A combined syndrome of juvenile polyposis and hereditary haemorrhagic teleangiectasia associated with mutations in MADH4 (SMAD4). Lancet 2004; 2004: 852-859.

2. Schwenter F, Faughnan ME, Gradinger AB, et al. Juvenile polyposis, hereditary hemorrhagic telangiectasia and early onset colorectal cancer in patients with SMAD4 mutation, J Gastroenterol 2012; 47: 795-804.

3. Cohen S, Hyer W, Mas E, et al. Management of Juvenile Polyposis Syndrome in Children and Adolescents: A Position Paper From the ESPGHAN Polyposis Working Group, JPGN, 2019; 68.

4. Jass JR, Wiliams CB, Bussey HJ, et al. Juvenil polyposis - a precancerous condition. Histopathology 1988; 13: 619-630.

5. Wain KE, Ellingson MS, McDonald J, et al. Appreciating the broad clinical features of SMAD4 mutation carriers: a multicenter chart review. Genet Med 2014; 16: 588-593.

6. Haidle JL, Howe JR. Juvenile Polyposis Syndrome. In: Adam MP, Ardinger HH, Pagon RA, et al. Editors. GeneReviews. University of Washington, Seattle; 1993-2018.
7. Shovlin CL, Guttmacher AE, Buscarini E, et al. Diagnostic criteria for hereditary hemorrhagic telangiectasia (Rendu-Osler-Weber syndrome). Am J Med Genet 2000; 91: 66-67.

8. Jelsig AM, Torring PM, Kjeldsen AD, et al. JP-HHT phenotype in Danish patients with SMAD4 mutations. Clin Genet 2016; 90: 55-62.

9. Aretz S, Stienen D, Uhlhaas S, et al. High proportion of large genomic deletions and a genotype-phenotype update in 80 unrelated families with juvenile polyposis syndrome. J Med Genet 2007; 44: 702-709.

10. Teekakirikul P, Milewicz DM, Miller DT, et al. Thoracic Aortic Disease in Two Patients With Juvenile Polyposis Syndrome and SMAD4 Mutations. Am J Med Genet Part A 2013; 161A: 185-191.

11. Iyer NK, Burke CA, Leach BH, et al. SMAD4 mutation and the combined syndrome of juvenile polyposis syndrome and hereditary heamorrhagic telangiectasia. Thorax 2010; 65: 745-746.

12. Chung AD, Mortele KJ. Combined juvenile polyposis syndrome and hereditary hemorrhagic telangiectasia (JPS/HHT) with MRI and endoscopic correlation. Clin Imaging 2019; 54: 37-39.

13. Pantaleoni J, Parsapour K, Kon AA. Inhalated nitric oxide and sildenafil therapy for a pediatric patient with multiple pulmonary arteriovenosus malformations. J Pediatr Intensive Care 2013; 2: 181-185.

14. Williams JCB, Hamilton JK, Shiller M, et al. Combined juvenile polyposis and hereditary hemorrhagic telangiectasia, Proc (Bayl Univ Med Cent) 2012; 25: 360-364.

15. Schwenter F, Faughnan ME, Gradinger AB, et al. Juvenile polyposis, hereditary hemorrhagic telangiectasia, and early onset colorectal cancer in patients with SMAD4 mutation. J Gastroenetrol 2012; 47: 795-804. 